\title{
Diversidade alimentar de crianças indígenas de dois municípios da Amazônia Ocidental brasileira
}

\author{
Food diversity among indigenous children from two municipalities \\ of the Brazilian Western Amazon
}

Vanizia Barboza da Silva Maciel (https://orcid.org/0000-0002-1166-7551) 1

Kelly Pereira Coca (https://orcid.org/0000-0002-3604-852X) 2

Lucíola Sant'Anna de Castro (https://orcid.org/0000-0001-9729-8306) 3

Ana Cristina Freitas de Vilhena Abrão (https://orcid.org/0000-0001-6249-2467) 2

${ }^{1}$ Centro Multidisciplinar, Universidade Federal do Acre. Estrada do Canela Fina $\mathrm{Km} 12$, Campus Floresta. 69980-000 Cruzeiro do Sul AC Brasil. enfer_van@yahoo.com.br ${ }^{2}$ Departamento de Enfermagem na Saúde da Mulher, Escola Paulista de Enfermagem, Universidade Federal de São Paulo. São Paulo SP Brasil.

${ }^{3}$ Departamento de Medicina Preventiva, Universidade Federal de São Paulo. São Paulo SP Brasil. São Paulo SP Brasil.

\begin{abstract}
Our aim was to explore the feeding practices of indigenous children younger than two years old, living in Cruzeiro do Sul and Mâncio Lima, in Acre, Brazil. A cross-sectional study was conducted with indigenous children of the Katukina (Cruzeiro do Sul), Nukini, Nawa and Poyanawa (Mâncio Lima) ethnicities in October 2013. We used a structured questionnaire on the food consumption of the day before the collection, based on the food indicators proposed by the Ministry of Health. A total of 94 children (50\% Katukina, 27\% Poyanawa, $13 \%$ Nukini, and 10\% Nawa) were studied. Among children under 6 months of age, although the majority was breastfed, $42.1 \%$ drank water, $15.8 \%$, savory food, and $11.1 \%$, local cuisine. In the age groups of 6 to 12 months and 13 to 23 months, a high consumption of ultra-processed foods was identified (52.6\% and $28.6 \%$ respectively), while the consumption of complementary food was insufficient (33\% of fruits and 25\% of savory food between 6 and 13 months, and $41.2 \%$ of fruits and $19.6 \%$ of savory food between 13 and 23 months). The feeding profile of these indigenous children was inadequate for their age, revealing a concerning situation for this population.
\end{abstract}

Keywords Infant nutrition, Feeding behavior, Indigenous population, Child health, Indians South American
Resumo Objetivou-se explorar a prática alimentar das crianças indígenas de zero a dois anos, residentes nos municípios de Cruzeiro do Sul e Mâncio Lima no Acre. Realizou-se estudo transversal com crianças indígenas das etnias Katukina (Cruzeiro do Sul), Nukini, Nawa e Poyanawa (Mâncio Lima) em outubro de 2013. Utilizou-se um questionário estruturado sobre o consumo alimentar do dia anterior à coleta, baseado nos indicadores alimentares propostos pelo Ministério da Saúde. Estudou-se 94 crianças (50\% da etnia Katukina, 27\% Poyanawa, 13\% Nukini e 10\% da Nawa). As crianças menores de 6 meses de idade, apesar da maioria receber leite materno, $42,1 \%$ delas consumiam água, 15,8\% comida de sal e 11,1\% preparados típicos da região. Nas faixas de idade de 6 a 12 meses e 13 a 23 meses identificou-se um alto consumo de alimentos ultraprocessados $(52,6 \% e$ $28,6 \%$ respectivamente) enquanto que o consumo de alimentos complementares relevou-se insuficiente (33\% de frutas e $25 \%$ de comida de sal na faixa de 6 a 13 meses e $41,2 \%$ de frutas e 19,6\% de comida de sal na faixa de 13 a 23 meses). O perfil alimentar das crianças indígenas mostrou-se inadequado para a idade, refletindo uma situação preocupante na saúde infantil desta população.

Palavras-chave Nutrição do lactente, População indigena, Comportamento alimentar, Saúde da criança, Índios sul-americanos 


\section{Introdução}

A prática alimentar de crianças menores de 2 anos no Brasil tem sido considerada um problema de saúde pública, devido à baixa prevalência de aleitamento materno (AM) e a introdução precoce de alimentos não saudáveis ${ }^{1}$. Esta situação estende-se para a população indígena do país que, apesar de apresentarem uma média de 5,1 meses de aleitamento materno exclusivo (AME), o aleitamento complementado (AMC) tem sido o mais frequentemente praticado $(73,7 \%)$ entre crianças das cidades indígenas de Cruzeiro do $\mathrm{Sul}^{2}$. O inadequado padrão alimentar é verificado na elevada prevalência de desnutrição $(25,7 \%$ de nanismo), revelado em inquérito nacional com 6.050 crianças indígenas ${ }^{3}$. O estudo também mostrou que a prevalência de desnutrição esteve associada às variáveis socioeconômicas como renda, escolaridade e acesso a serviços de saneamento, no entanto, o aleitamento teve efeito protetor para baixo peso entre crianças com até 12 meses de vida ${ }^{3-4}$.

Tradicionalmente os povos indígenas dedicam a maior parte de seu tempo em atividades voltadas para a alimentação ${ }^{5}$. O plantio, a colheita, a caça e a pesca são atividades diárias que embasam a alimentação do grupo, de acordo com a região em que eles vivem ${ }^{5}$. No entanto, o contato com os indivíduos não índios vem acelerando um processo complexo de mudanças entre as populações indígenas, principalmente no tocante ao perfil de saúde e nutrição $0^{6}$. A sociodiversidade indígena no Brasil, associada às desigualdades sociais em saúde, teve impacto na elevada prevalência da desnutrição infantil, anemia ferropriva, doenças parasitárias e infecciosas, aumentando a taxa de mortalidade de crianças indígenas no primeiro ano de vida ${ }^{6}$. A população indígena no Brasil apresenta altas prevalências de baixo peso ao nascer e déficit do crescimento, especialmente entre as crianças da região Norte do país, cujas famílias apresentavam precárias condições socioeconômicas e sanitárias e cujas mães eram anêmicas $^{3}$.

A alimentação infantil ameríndia, em algumas etnias, baseia-se no aleitamento materno durante o primeiro ano de vida, que muitas vezes se prolonga até os três anos de idade, aproximadamente ${ }^{7}$. A introdução de alimentos industrializados à dieta está relacionada ao número de adultos na família e ao nível socioeconômico, às condições ambientais de subsistência, e ao acesso aos centros urbanos ${ }^{7}$. Uma recente revisão sistemática da literatura, que buscou investigar o es- tado nutricional e a alimentação de crianças indígenas da América (México, Peru, Estados Unidos, Brasil e Guiana), mostrou que mesmo antes dos seis meses o mingau de mandioca começa a ser oferecido de forma esporádica e que, gradativamente, incluem na alimentação da criança: o peixe e algumas frutas, o açúcar refinado, biscoitos e guloseimas em geral ${ }^{7}$.

Apesar destas informações, poucos estudos na literatura investigaram as condições de alimentação e nutrição das populações indígenas no Brasil. A ausência de dados sobre a alimentação das crianças indígenas no Brasil e no estado do Acre reforça a importância de divulgar os dados desta pesquisa. Neste sentido, o objetivo deste artigo é identificar o padrão de consumo alimentar de crianças indígenas menores de 2 anos de idade.

\section{Método}

Trata-se de estudo transversal realizado com crianças indígenas habitantes das cidades de Cruzeiro do Sul (etnia Katukina) e Mâncio Lima (etnias Nukini, Nawa e Poyanawa), municípios do estado do Acre. A etnia Katukina está localizada às margens da BR 364, que liga a capital Rio Branco à Cruzeiro do Sul e outros municípios. Nas aldeias é preservada a língua Pano, e somente os homens falam a língua portuguesa quando estão fora da região $0^{8}$. O povo Nukini mora na região da Serra do Môa, um parque de preservação nacional; o idioma é o português, mas a Língua materna Pano é ensinada nas escolas. Já o povo Nawa habita a margem direita do Rio Môa, suas casas são tradicionais de moradores ribeirinhos e a comunicação é feita na língua portuguesa ${ }^{8}$. A etnia Poyanawa habita a margem direita do Rio Môa e suas malocas foram substituídas por casas tradicionais a partir do contato com os não índios ${ }^{8}$.

Segundo o Censo Demográfico de 2010, o número total de crianças indígenas com idade entre 0 e 2 anos era de 202 (104 na aldeia de Cruzeiro do Sul e 98 na de Mâncio Lima) ${ }^{9}$. Desta forma, o estudo foi planejado para entrevistar todas as crianças que estavam nas aldeias, durante o mês de visitação das regiões. Os critérios de exclusão foram: não encontrar a mãe ou responsável pela criança na residência após duas tentativas em períodos diferentes do dia na aldeia ou crianças cujas mães não aceitaram participar do estudo.

A coleta de dados foi realizada durante o mês de outubro de 2013, por uma das pesquisadoras, por meio de entrevistas que eram feitas com as 
mulheres durante as visitas diárias nas aldeias. Em algumas regiões ocorreu a necessidade de pernoitar nos locais devido ao tempo de viagem para acessar a região. A comunicação com as mulheres que não falavam a língua portuguesa foi realizada por meio da tradução simultânea da entrevista pelos agentes de saúde indígenas.

Os dados foram obtidos utilizando-se de um instrumento de coleta próprio com dados de caracterização da criança (etnia, idade atual, sexo, idade gestacional de nascimento, peso ao nascer e problemas de saúde desde o nascimento) e da mãe (idade, escolaridade, situação conjugal, ocupação, renda familiar e número de filhos).

O consumo alimentar das crianças foi avaliado a partir do formulário de marcadores de consumo alimentar para crianças menores de 6 meses e de 6 a 23 meses, disponível pelo Sistema de Vigilância Alimentar e Nutricional (SISVAN) do Ministério da Saúde do Brasil ${ }^{10}$. Para a avaliação das crianças de 6 a 23 meses foram consideradas as questões do primeiro bloco do formulário, que têm por objetivo caracterizar a introdução da alimentação complementar e identificar o tipo de alimentação atual. O formulário, que se refere ao consumo alimentar do dia anterior ao do inquérito, foi adaptado para conter um campo aberto que permitiu conhecer o consumo de outros alimentos pertencentes à cultura local.

Para realizar o diagnóstico da prática alimentar, os alimentos foram classificados em grupos de acordo com a faixa etária, a saber: leite (materno exclusivo e não exclusivo, e artificial), água, alimentos complementares (frutas e comida de sal) e outros alimentos para os menores de 6 meses; leite (materno e artificial), alimentos complementares (frutas e comida de sal) e outros alimentos para crianças de 6 a 23 meses. A categoria "outros alimentos" foi utilizada para incluir os preparados regionais que não puderam ser categorizados de acordo com o tipo de alimentação atual do Ministério da Saúde do Brasil ${ }^{10}$. Os demais alimentos relatados foram classificados de acordo com seu grau de processamento ${ }^{11}$.

$\mathrm{Na}$ descrição dos dados utilizaram-se medidas de tendência central (média e mediana) e de dispersão (desvio-padrão e valores mínimo e máximo) para variáveis quantitativas, e frequências relativas e absolutas para as de natureza qualitativa. Empregaram-se os testes estatísticos de Qui-quadrado de Pearson, o Exato de Fisher e o teste $t$ de Student, considerando o nível de significância $\alpha$ igual a $5 \%$.

Para as análises de associação entre as variáveis de consumo alimentar e o município de re- sidência das crianças, o número amostral variou de acordo com a quantidade de registros com dados completos.

A pesquisa foi aprovada pela Comissão $\mathrm{Na}$ cional de Ética e pelo Comitê de Ética da Universidade Federal de São Paulo, conforme Resoluções 466/2012 e 304/2000 do Conselho Nacional de Saúde (específica para a área de Povos Indígenas) como parte do projeto "Fatores associados ao desmame precoce em crianças indígenas de dois municípios da Amazônia Ocidental Brasileira" $^{2}$. O Termo de Consentimento Livre e Esclarecido (TCLE) foi obtido por meio da assinatura das mães ou da digital do polegar, quando não alfabetizada.

\section{Resultados}

A população do estudo foi composta por 94 crianças, a maioria da etnia Katukina $(48,9 \%)$ na cidade de Cruzeiro do Sul, seguido da Poyanawa $(28,7 \%)$, Nukini $(12,8 \%)$ e Nawa $(9,6 \%)$, na cidade de Mâncio Lima. A idade média das crianças foi de 12,7 meses $(\mathrm{DP}=6,4 ;$ mín. $=15$ dias, máx.= 23 meses); a maioria $(54,3 \%)$ tinha de 13 a 23 meses de idade, era do sexo masculino $(53,2 \%)$, nasceu com 37 semanas ou mais (98\%) e o peso ao nascer foi, em média, 3.238 gramas $(\mathrm{DP}=599 ;$ mín. $=1.500$ e máx.= 4.800);88\% das crianças apresentaram pelo menos um problema de saúde em algum momento da vida, sendo respiratório $(83 \%)$ e diarreia $(76 \%)$ os mais frequentes. Em relação às características maternas, a idade média das mães foi de 26 anos $(\mathrm{DP}=6,7$; mín. $=13$, máx. $=43$ ), a maioria possuía ensino fundamental incompleto $(73,4 \%)$, vivia com companheiro $(75,5 \%)$, apresentava uma média de 3 filhos $(\mathrm{DP}=2,2$; mín.=1, máx.=10), era do lar $(82,4 \%)$ e apresentava renda menor do que um salário-mínimo nacional (78\%).

No que se refere à alimentação, a Tabela 1 mostra os dados alimentares das crianças com até seis meses de idade, de acordo com o município das etnias. Apesar da maioria receber leite materno $(89,5 \%)$ e estar em AME $(52,6 \%)$, $42,1 \%$ delas consumiam água e $15,8 \%$ comida de sal. Verificou-se que as crianças menores de seis meses, cuja etnia é localizada no município de Mâncio Lima, apresentaram maior consumo de água $(\mathrm{p}=0,020)$ quando comparadas às de etnia localizada em Cruzeiro do Sul.

Em relação às crianças de 6 a 12 meses, a maioria recebeu leite materno $(79,2 \%)$, no entanto, os percentuais de consumo de alimentos 
complementares foram baixos $(33,3 \%$ frutas e $25 \%$ comida de sal), especialmente no município de Cruzeiro do Sul (0\% comida de sal). O consumo de água foi significativamente menor nesta região $(\mathrm{p}=0,010)$, enquanto que alimento ultraprocessado foi consumido pela maioria na população geral entre crianças com 6 a 12 meses $(52,6 \%)$ (Tabela 2$)$.

$\mathrm{Na}$ faixa de idade entre 13 e 23 meses, menos da metade $(37,3 \%)$ das crianças estavam recebendo leite materno, com maior presença entre aquelas etnias localizadas em Mâncio Lima $(\mathrm{p}=0,009)$, no entanto, quase $1 / 3$ delas consumiram alimentos ultraprocessados $(28,6 \%$, $\mathrm{p}<0,001)$ e a grande maioria não consumiu alimentos complementares $(19,6 \%$ de comida de sal, $\mathrm{p}<0,001$ ) (Tabela 3 ).

Quanto à caracterização de outros alimentos consumidos e seu preparo, observou-se que na etnia Katukina a alimentação baseava-se no consumo da banana, farinha e macaxeira. Na região chamada de "banana grande", há grandes plantios da banana, segundo alimento mais utilizado pelas crianças, consumido em um preparado chamado de "mingau". A macaxeira, produto típico da região, era consumido sozinho, cozido ou acompanhado de outros alimentos, e o preparo mais comum era em forma de farinha ou massa

Tabela 1. Frequências absolutas e relativas percentuais do consumo de alimentos de crianças menores $<6$ meses, segundo o município das etnias.

\begin{tabular}{|c|c|c|c|c|c|c|c|}
\hline \multirow{2}{*}{ Alimentos } & \multicolumn{2}{|c|}{ Mâncio Lima } & \multicolumn{2}{|c|}{ Cruzeiro do Sul } & \multicolumn{2}{|c|}{ Total } & \multirow[b]{2}{*}{$\mathbf{p}$} \\
\hline & $\mathbf{n}$ & $\%$ & $\mathbf{n}$ & $\%$ & $\mathbf{n}$ & $\%$ & \\
\hline \multicolumn{8}{|l|}{ Leite } \\
\hline AME & 3 & 30,0 & 7 & 77,8 & 10 & 52,6 & $0,070^{\mathrm{b}}$ \\
\hline Leite materno & 8 & 80,0 & 9 & 100 & 17 & 89,5 & $0,474^{\mathrm{a}}$ \\
\hline Leite artificial & 1 & 10,0 & 0 & 0 & 1 & 5,3 & $>0,999^{b}$ \\
\hline Água & 7 & 70,0 & 1 & 11,1 & 8 & 42,1 & $0,020^{\mathrm{b}}$ \\
\hline \multicolumn{8}{|l|}{ Al. complementares } \\
\hline Frutas & 0 & 0 & 1 & 11,1 & 1 & 5,3 & $0,474^{\mathrm{b}}$ \\
\hline Comida de sal & 2 & 20,0 & 1 & 11,1 & 3 & 15,8 & $>0,999^{b}$ \\
\hline Outros alimentos & 1 & 11,1 & 1 & 11,1 & 2 & 11,1 & $>0,999^{\mathrm{b}}$ \\
\hline
\end{tabular}

Fonte: Elaborada pelas autoras.

Tabela 2. Frequências absolutas e relativas percentuais do consumo de alimentos de crianças entre 6 e 12 meses, segundo o município das etnias.

\begin{tabular}{|c|c|c|c|c|c|c|c|}
\hline \multirow{2}{*}{ Alimentos } & \multicolumn{2}{|c|}{ Mâncio Lima } & \multicolumn{2}{|c|}{ Cruzeiro do Sul } & \multicolumn{2}{|c|}{ Total } & \multirow{2}{*}{$\mathbf{p}$} \\
\hline & $\mathrm{n}$ & $\%$ & $\mathbf{n}$ & $\%$ & $\mathbf{n}$ & $\%$ & \\
\hline \multicolumn{8}{|l|}{ Leite } \\
\hline Leite materno & 13 & 72,2 & 6 & 100,0 & 19 & 79,2 & $0,280^{\mathrm{a}}$ \\
\hline Leite artificial & 7 & 38,9 & 2 & 33,3 & 9 & 37,5 & $>0,999^{b}$ \\
\hline Àgua & 18 & 100 & 3 & 50,0 & 21 & 87,5 & $0,010^{\mathrm{b}}$ \\
\hline \multicolumn{8}{|l|}{ Al. complementares } \\
\hline Fruta & 6 & 33,3 & 2 & 33,3 & 8 & 33,3 & $>0,999^{b}$ \\
\hline Comida de sal & 6 & 33,3 & 0 & 0 & 6 & 25,0 & $0,277^{\mathrm{b}}$ \\
\hline Outros alimentos & 14 & 87,5 & 4 & 66,7 & 18 & 81,8 & $0,292^{b}$ \\
\hline Ultraprocessados $^{*}$ & 9 & 60,0 & 1 & 25,0 & 10 & 52,6 & $0,303^{b}$ \\
\hline
\end{tabular}

aui-quadrado de Person; ${ }^{b}$ Teste Exato de Fisher. Al. complementares - Alimentos complementares. ${ }^{*}$ Formulações industriais feitas principalmente de substâncias extraídas de alimentos, derivadas de constituintes de alimentos ou produzidas em laboratório com base em matérias orgânicas como petróleo e carvão ${ }^{11}$. 
Tabela 3. Frequências absolutas e relativas percentuais do consumo de alimentos de crianças entre 13 e 23 meses, segundo o município das etnias.

\begin{tabular}{|c|c|c|c|c|c|c|c|}
\hline \multirow{2}{*}{ Alimentos } & \multicolumn{2}{|c|}{ Mâncio Lima } & \multicolumn{2}{|c|}{ Cruzeiro do Sul } & \multicolumn{2}{|c|}{ Total } & \multirow{2}{*}{$\mathbf{p}$} \\
\hline & $\mathbf{n}$ & $\%$ & $\mathbf{n}$ & $\%$ & $\mathbf{n}$ & $\%$ & \\
\hline \multicolumn{8}{|l|}{ Leite } \\
\hline Leite materno & 12 & 60,0 & 7 & 22,6 & 19 & 37,3 & $0,009^{a}$ \\
\hline Leite artificial & 4 & 20,0 & 2 & 6,5 & 6 & 11,8 & $0,195^{\mathrm{b}}$ \\
\hline Água & 19 & 95,0 & 28 & 90,3 & 47 & 92,2 & $>0,999^{a}$ \\
\hline \multicolumn{8}{|l|}{ Al. complementares } \\
\hline Fruta & 9 & 45,0 & 12 & 38,7 & 21 & 41,2 & $0,773^{a}$ \\
\hline Comida de sal & 9 & 45,0 & 1 & 3,2 & 10 & 19,6 & $<0,001^{\mathrm{b}}$ \\
\hline Outros alimentos & 19 & 100 & 30 & 96,8 & 49 & 98,0 & $>0,999^{a}$ \\
\hline Ultraprocessados ${ }^{*}$ & 13 & 68,4 & 1 & 3,3 & 14 & 28,6 & $<0,001^{\mathrm{b}}$ \\
\hline
\end{tabular}

${ }^{a}$ Qui-quadrado de Person; bTeste Exato de Fisher. Al. complementares - alimentos complementares. ${ }^{*}$ Formulações industriais feitas principalmente de substâncias extraídas de alimentos, derivadas de constituintes de alimentos ou produzidas em laboratório com base em matérias orgânicas como petróleo e carvão ${ }^{11}$.

Fonte: Elaborada pelas autoras.

(goma) para fazer tapioca e outros. A farinha é consumida juntamente com outros alimentos ou mesmo sozinha.

Nas etnias de Nawa e de Nukini, destacamse o frequente consumo de água, mesmo entre crianças em AM menores de 6 meses. O mingau de massa de mandioca é consumido como principal alimento, e trata-se de um preparo típico da região para alimentar as crianças, oriundo da macaxeira. Entre carnes consumidas, observam-se consumo de carne de caça (tatu e cutia). Caracterizadas como comunidades ribeirinhas e de localização próxima do rio, observa-se maior frequência de consumo de produtos industrializados.

Em relação à etnia Poyanawa, a alimentação comumente preparada é chamada de "pirão", alimento típico regional que consiste em uma mistura de farinha de mandioca com o caldo da carne, frango ou peixe. As crianças consomem alimentos da família, que envolve uma quantidade significativa de produtos industrializados (biscoito, suco industrializado, iogurte, enlatados, macarrão instantâneo, frango e carne bovina industrializados), além de arroz e feijão.

No que se refere aos alimentos ultraprocessados, a maior prevalência de consumo foi observada foi entre as crianças de Mâncio Lima, especialmente na faixa etária entre 13 e 23 meses de idade $(68,4 \%, \mathrm{p}<0,001)$ (Tabela 3$)$. Os tipos de ultraprocessados mais consumidos estavam o biscoito $(65,2 \%)$ e suco artificial $(58,3 \%)$ (dados não apresentados em tabela).

\section{Discussão}

O estudo mostrou que a amamentação das crianças de 0 a 6 meses está comprometida com a introdução precoce de alimentos que não o leite materno. Já as crianças entre 7 e 24 meses apresentaram uma baixa diversidade de alimentos em sua refeição, sendo o alimento ultraprocessado muito presente na alimentação destas crianças, especialmente entre 6 e 12 meses.

De acordo com a Organização Mundial da Saúde, o leite materno é suficiente para atender as necessidades nutricionais da criança até os seis meses de idade, sem chás ou água ${ }^{12}$. Após este período, recomenda-se a introdução gradativa de alimentos saudáveis concomitante com o aleitamento materno até dois anos ou mais ${ }^{12}$. De acordo com os dez passos para uma alimentação saudável do Brasil ${ }^{10}$, a recomendação da alimentação da criança inclui alimentos variados e de importância nutricional, incluindo cereais/tubérculos, carnes, leguminosas, frutas, legumes e verduras.

Sabe-se que a prática alimentar de uma nação é influenciada pela disponibilidade dos alimentos da região e cultura de seu preparo e consumo. Nas florestas tropicais, o uso de seus recursos caracteriza tradicionalmente uma dieta diversificada. A colonização das regiões teve influência no comportamento das famílias indígenas, que passaram a transitar do hábito comunitário para a economia de mercado ${ }^{13}$. As famílias indígenas da região de São Gabriel da Cachoeira, no Noroeste da Amazônia brasileira articulam os recursos 
produzidos na floresta para compor hábitos alimentares da população, sendo a diversidade alimentar dependente da territorialidade do grupo doméstico, no entanto, notou-se uma mudança de hábitos alimentares, decorrente do desenvolvimento do extrativismo, correspondendo às práticas de atividades remuneradas como a extração de recursos locais ${ }^{13}$. A diversidade alimentar também tem sido observada e pouco praticada em crianças não indígenas da região Sudeste do Brasil, sugerindo que as políticas públicas voltadas à alimentação das crianças menores de dois anos sejam intensificadas ${ }^{14}$.

Na região do Cruzeiro do Sul, a pavimentação da BR 364, a partir de 2000, provocou mudança significativa na dieta alimentar dos Katukinas, pois a estrada afugentou os animais de caça, e deu oportunidade aos caçadores profissionais e comprometeu suas plantações, roçados apenas como banana, macaxeira, batata-doce e mamão. Além disso, as habitações mudaram para casas que precisam de segurança como paredes e trancas em suas moradias ${ }^{8}$. Já a região de Mâncio Lima, as etnias vivem do plantio e caça. A etnia Nukini cria animais (porco, galinha, pato, cabras e ovelhas) tanto para consumo como para o comércio, somado ao cultivo de produtos agrícolas (milho, arroz, macaxeira, feijão, cana-de-açúcar, tabaco e inhame) e plantio e coleta de frutas da floresta (açaí, a abacaba, o buriti, o patuá e a pupunha). A base da alimentação dos Nawas é a mandioca complementada com caça e pesca, plantam cana-de-açúcar, pupunha e abacaxi e cultivam temperos, plantas medicinais e árvores frutíferas $^{8}$. A etnia Poyanawa preserva a atividade de subsistência baseada na agricultura familiar com o plantio (arroz, batata-doce, inhame, mandioca, milho e frutas) e praticam a piscicultura como alternativa econômica sustentável ${ }^{8}$. Os produtos industrializados, que também fazem parte da dieta alimentar são adquiridos no centro da cidade de Mâncio Limå

Neste sentido, o presente estudo identificou a presença de hábitos alimentares não tradicionais misturados com a dieta da cultura regional entre as crianças residentes nos municípios de Cruzeiro do Sul e Mâncio Lima no Acre. Esta transição dos hábitos alimentares também é característica observada entre os indígenas do Alto Xingu no Mato Grosso como, por exemplo, o uso de açúcar refinado, biscoitos e guloseimas em ge$\mathrm{ral}^{15}$. A adequação nutricional das dietas nativas predominam para a ocidentalização dos hábitos alimentares, com a introdução de itens como sal, açúcar, produtos industrializados e refinados ${ }^{16}$.
Em termos gerais, o quadro usualmente descrito se caracteriza pela redução da diversidade alimentar, com o aumento do consumo de açúcares simples e gorduras, concomitantemente à redução da ingestão de fibras e micronutrientes ${ }^{16}$. O mesmo ocorreu no estudo realizado por Coelho et al. ${ }^{17}$, com crianças não indígenas menores de 24 meses e utilizando os formulários de marcadores de consumo alimentar do SISVAN ${ }^{11}$, em que mostrou que $55,4 \%$ das crianças avaliadas apresentaram comportamento de risco para obesidade, como por exemplo o consumo de suco industrializado e refrigerante.

O consumo de alimentos processados, tais como açúcar, frituras, refrigerantes, balas, salgadinhos, biscoitos recheados dentre outros devem ser evitados especialmente nos primeiros anos de vida $a^{10}$. O consumo desse tipo de alimento está associado ao excesso de peso e à obesidade na infância, que reflete no desenvolvimento de várias doenças alérgicas, metabólicas e cardiovasculares $^{18}$.

Assim como o tipo do alimento consumido, a diversidade alimentar também é importante para o crescimento e desenvolvimento saudável da criança ${ }^{10}$, condição pouco observada na prática alimentar habitual das crianças das etnias do presente estudo. A base da dieta dos ameríndios da Amazônia é a banana e mandioca; a proteína consumida é advinda da caça (peixes e animais silvestres), quando presente ${ }^{19}$. Desta forma, a alimentação das crianças é considerada pobre em densidade calórica que, provavelmente, seria a responsável pelo atraso no crescimento e estado nutricional deficiente, observado entre as crianças indígenas ${ }^{19}$. O mesmo ou pior padrão alimentar foi observado na população estudada, na qual verificou-se insuficiente consumo de alimentos com adequada densidade nutricional e a presença daqueles considerados não saudáveis para o desenvolvimento infantil. Esses achados são semelhantes ao perfil epidemiológico nutricional avaliado em outras etnias no Brasil, como é o caso da comunidade Wari', povo indígena localizado em Rondônia, no Sudoeste amazônico, cujas prevalências de baixa estatura $(61,7 \%)$ e peso $(51,7 \%)$ de crianças menores de cinco anos estão entre as mais elevadas entre as populações indígenas no Brasil ${ }^{20}$. O déficit de estatura também foi identificado nas crianças Guarani menores de 5 anos, nos estados do Rio de Janeiro e São Paulo, 96\% maior do que nas crianças indígenas do Brasil $(25,7 \%)^{21}$. Este estudo destaca elevadas prevalências de desnutrição crônica e anemia na criança Guarani, e põe em evidência as graves 
desigualdades em saúde e nutrição que afetam a criança indígena no país ${ }^{21}$.

Apesar da avaliação do estado nutricional não ter sido objeto deste estudo, o déficit nutricional observado em crianças indígenas, em todas as faixas etárias a partir do sexto mês de vida, das comunidades Kaiowá e Guarani, área indígena de Caarapó, Mato Grosso do Sul, foi influenciado pelo baixo nível educacional, precárias condições socioeconômicas, ambientais e de saúde desse povo $^{22}$, situações estas, observadas nas etnias localizadas em Mâncio Lima e Cruzeiro do Sul. Um estudo na comunidade indígena Teréna, Mato Grosso do Sul evidenciou resultado concordante, no qual a prevalência de desnutrição infantil estava elevada, superior à identificada em comunidade indígena urbana e área rural da região Centro-Oeste, porém inferior às reportadas por grupos indígenas da região amazônica, mais frequente quando pertenciam à família de menor renda per capita, ao sexo masculino e na faixa etária de 6 a 23 meses de idade ${ }^{23}$.

Desta forma, a alimentação das crianças indígenas reflete situação preocupante na saúde infantil desta população e os achados poderão indicar estratégias para refletir e discutir junto à população indígena. Dentre as limitações do estudo, observaram os numerosos documentos para adqui- rir as autorizações para acessar as terras indígenas, bem como as autorizações ético-legais, pela característica da população estudada. Tais condições demandaram tempo significativo prévio à coleta dos dados. A falta de dados atuais no Sistema de Informação da Atenção à Saúde Indígena (SIASI), Fundação Nacional de Saúde (FUNASA) / MS, dificultou o conhecimento do real número de crianças na faixa etária do estudo, permitindo atingir cerca de 50\% do previsto. Destaca-se ainda que o acesso às comunidades indígenas limitou abrangência de outras aldeias indígenas da região, pois além de distante o acesso estava na dependência do nível da água do rio, que poderia estar cheio ou vazio no período da "estiagem" da Amazônia ou mesmo a necessidade de transporte aéreo.

\section{Conclusão}

Foi identificado que a alimentação das crianças indígenas é constituída por alimentos regionais e por produtos alimentícios ultraprocessados. Os alimentos de má qualidade nutricional e a pouca diversidade refletem a atual situação alimentar das crianças indígenas menores de 2 anos residentes nos municípios de Cruzeiro do Sul e Mâncio Lima no Acre.

\section{Colaboradores}

VBS Maciel e ACFV Abrão participaram na concepção, delineamento, análise e interpretação dos dados, da redação do manuscrito, da revisão crítica e aprovação da versão final do artigo. KP Coca e LS Castro participaram da análise e interpretação dos dados, da redação do manuscrito, da revisão crítica e aprovação da versão final do artigo.

\section{Agradecimentos}

Às lideranças indígenas que autorizaram nossa entrada nas suas etnias, aos agentes indígenas de saúde que contribuíram em todas as entrevistas com a mediação da conversa e tradução, à FUNAI, que autorizou nossa entrada em terras indígenas e contribuiu muito com nossa pesquisa e às mulheres indígenas que aceitaram participar do estudo. 


\section{Referências}

1. Venancio SI, Escuder MM, Saldiva SR, Giugliani ER. Breastfeeding practice in the Brazilian capital cities and the Federal District: current status and advances. J Pediatr (Rio J). 2010;86(4): 317-324.

2. Maciel VBS, Silva RPM, Sañudo A, Abuchaim ESV, Abrão ACFV. Aleitamento materno em crianças indígenas de dois municípios da Amazônia Ocidental Brasileira. Act paul Enferm 2016; 29: 469-475.

3. Horta BL, Santos RV, Welch JR, Cardoso AM, Santos JV, Assis AM, Lira PCI, Coimbra Jr CEA. Nutritional status of indigenous children: findings from the First National Survey of Indigenous People's Health and Nutrition in Brazil. Int J Equity Health 2013;12:23.

4. Coimbra EACJ. Health and indigenous peoples in Brazil: reflections based on the First National Survey of Indigenous People's Health and Nutrition. Cad Saude Publica 2014; 30(4):855-859.

5. de Araujo MLL, Kubo RR. Segurança alimentar e nutricional e povos indígenas: a experiência dos asheninkas do alto rio envira com o programa de aquisição de alimentos (PAA). Rev Parana Desenvolv 2017; 38(132):195-210.

6. Coimbra CE, Santos RV, Welch JR, Cardoso AM, de Souza MC, Garnelo L, Rassi E, Follér M-L, Horta BL . The first national survey of indigenous people's health and nutrition in Brazil: rationale, methodology, and overview of results. BMC Public Health 2013;13:52.

7. Silva LM, Silva IA. O estado nutricional e a alimentação infantil ameríndia: uma revisão sistemática de literatura. Revista Iberoamericana de Educación e Investigación en Enfermería 2014; 4(2):41-53.

8. Governo do Estado do Acre. Fundação de Cultura e Comunicação Elias Mansour. Povos Indigenas no Acre. Brasil: Governo do Estado do Acre; 2010.

9. Brasil. Ministério da Saúde (MS). Fundação Nacional de Saúde (FUNASA). Sistema de informação da Atenção à Saúde Indígena (SIASI). Brasília: MS; FUNASA; 2013.

10. Brasil. Ministério da Saúde (MS). Secretaria de Atenção à Saúde. Departamento de Atenção Básica. Orientações para avaliação de marcadores de consumo alimentar na atenção básica [recurso eletrônico] / MS, Secretaria de Atenção à Saúde, Departamento de Atenção Básica. Brasília: MS; 2015.

11. Brasil. Ministério da Saúde (MS). Secretaria de Atenção à Saúde. Guia alimentar para a população brasileira / Ministério da Saúde. Secretaria de Atenção à Saúde. Departamento de Atenção Básica. Brasília: MS; 2014. 156p.

12. World Health Organization (WHO), United States Agency for International Development (USAID). Fundo das Nações Unidas para a Infância (UNICEF). Indicators for assessing infant and young child feeding practices: conclusions of a consensus meeting held 6-8 November 2007 in Washingnton, DC, USA. Geneva: WHO; 2008.

13. Eloy L. Diversidade alimentar e urbanização: o papel das migrações circulares indígenas no Noroeste Amazônico Anthropology of food. 2009; S6.
14. Oliveira MICd, Rigotti RR, Boccolini CS. Fatores associados à falta de diversidade alimentar no segundo semestre de vida. Cad Saude Coletiva 2017; 25:65-72.

15. Mondini L, Rodrigues DA, Gimeno SGA, Baruzzi RG. Nutritional status and hemoglobin values of Aruak and Karibe Indian children - Upper Xingu, Central Brazil, 2001-2002. Rev Bras Epidemiol 2009; 12(3):469-477.

16. Castro TG, Schuch I, Conde WL, Veiga J, Leite MS, Dutra CL, Zuchinali P, Barufaldi LA. Nutritional status of Kaingang Indians enrolled in 12 indigenous schools in the State of Rio Grande do Sul, Brazil. Cad Saude Publica 2010; 26(9):1766-1776.

17. Coelho LdC, Asakura L, Sachs A, Erbert I, Novaes CdRL, Gimeno SGA. Food and Nutrition Surveillance System/SISVAN: getting to know the feeding habits of infants under 24 months of age. Cien Saude Coletiva 2015;20:727-738.

18. Elenberg Y, Shaoul R. The role of infant nutrition in the prevention of future disease. Front Pediatr 2014; 2:73.

19. Dufour DL. Diet and nutritional status of Ameridians: a review of the literature. Cad Saude Publica. 1991; 7(4):481-502.

20. Leite MS, Santos RV, Coimbra CE, Jr. Seasonality and nutritional status of indigenous peoples: the case of Wari' in Rondonia State, Brazil. Cad Saude Publica 2007; 23(11):2631-2642.

21. Barreto CT, Cardoso AM, Coimbra CE, Jr. Nutritional status of Guarani indigenous children in the States of Rio de Janeiro and Sao Paulo, Brazil. Cad Saude Publica 2014; 30(3):657-662.

22. Picoli RP, Carandina L, Ribas DL. Mother-child health and nutrition of Kaiowa and Guarani indigenous children, Caarapo Reserve, Mato Grosso do Sul, Brazil. Cad Saude Publica 2006; 22(1):223-227.

23. Ribas DL, Sganzerla A, Zorzatto JR, Philippi ST. Child health and nutrition in a Terena indigenous community, Mato Grosso do Sul, Brazil. Cad Saude Publica 2001; 17(2):323-231.

Artigo apresentado em 07/02/2019

Aprovado em 16/09/2019

Versão final apresentada em 18/09/2019

Editores-chefes: Romeu Gomes, Antônio Augusto Moura da Silva 